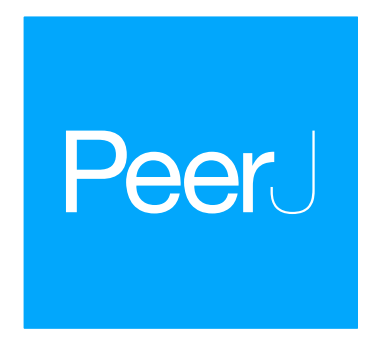

\title{
Evaluation of DESS as a storage medium for microbial community analysis
}

\author{
Kevin M. Lee, Madison Adams and Jonathan L. Klassen \\ Department of Molecular and Cell Biology, University of Connecticut, Storrs, CT, USA
}

\section{ABSTRACT}

Microbial ecology research requires sampling strategies that accurately represent the microbial community under study. These communities must typically be transported from the collection location to the laboratory and then stored until they can be processed. However, there is a lack of consensus on how best to preserve microbial communities during transport and storage. Here, we evaluated dimethyl sulfoxide, ethylenediamine tetraacetic acid, saturated salt (DESS) solution as a broadly applicable preservative for microbial ecology experiments. We stored fungus gardens grown by the ant Trachymyrmex septentrionalis in DESS, 15\% glycerol, and phosphate buffered saline (PBS) to test their impact on the fungus garden microbial community. Variation in microbial community structure due to differences in preservative type was minimal when compared to variation between ant colonies. Additionally, DESS preserved the structure of a defined mock community more faithfully than either $15 \%$ glycerol or PBS. DESS is inexpensive, easy to transport, and effective in preserving microbial community structure. We therefore conclude that DESS is a valuable preservative for use in microbial ecology research.

Submitted 12 July 2018

Accepted 24 December 2018

Published 5 February 2019

Corresponding author

Jonathan L. Klassen,

jonathan.klassen@uconn.edu

Academic editor

Michael LaMontagne

Additional Information and Declarations can be found on page 11

DOI 10.7717/peerj.6414

(c) Copyright

2019 Lee et al.

Distributed under

Creative Commons CC-BY 4.0

OPEN ACCESS
Subjects Ecology, Microbiology

Keywords DESS, Microbiome, Microbial ecology, Preservative, Fungus-growing ants

\section{INTRODUCTION}

Microbial ecologists frequently use culture-independent methods to study the structure of microbial communities (Hugerth \& Andersson, 2017). Such experiments face many design challenges that must be considered so that data they produce matches the structure of the original microbial community (Vandeputte et al., 2017; Pollock et al., 2018). Sample collection, handling, and storage are the first steps in any culture-independent study, and decisions made during this phase of a study can strongly affect downstream analyses (Hugerth \& Andersson, 2017; Vandeputte et al., 2017). Ideally, DNA should be extracted from samples immediately following collection (Rochelle et al., 1994; Cardona et al., 2012). However, sampling typically occurs outside of the laboratory and samples can therefore experience prolonged and sometimes poorly controlled transportation and storage conditions that permit nucleic acid degradation and/or microbial growth (Amir et al., 2017). Either of these processes will alter the structure of the sampled microbial community such that obtained experimental data does not match the original structure of that community.

Cold storage (at $-80,-20$, or $4{ }^{\circ} \mathrm{C}$, in order of preference) is the accepted gold standard for protecting samples from potentially adverse conditions that compromise microbial 
community structure (Rissanen et al., 2010; Williamson et al., 2011; Choo, Leong \& Rogers, 2015; Hale et al., 2016; Kia et al., 2016; Song et al., 2016, but see also Bahl, Bergström \& Licht, 2012). One disadvantage of cold storage is that cold chains are fragile, especially when collection sites are remote (Vandeputte et al., 2017). Electrically-powered cooling requires substantial and robust physical infrastructure. Alternatively, refrigerants such as ice, dry ice, and liquid nitrogen can be difficult to obtain, must be refreshed routinely, and (in the case of dry ice and liquid nitrogen) are regulated or prohibited on many modes of transportation. These issues limit the deployment and robustness of cold storage for preserving microbial community samples, despite its effectiveness.

Many studies have used preservative media as an alternative solution to overcome the logistical difficulties of cold transport and storage. These include proprietary media such as DNAgard, RNAlater, OMNIgene.GUT, and LifeGuard, and less-expensive non-proprietary media such as Tris-EDTA, ethanol, and phenol-chloroform (Rissanen et al., 2010; Gaither et al., 2011; Gray, Pratte \& Kellogg, 2013; Tatangelo et al., 2014; Choo, Leong \& Rogers, 2015). As with cold storage, these buffers attempt to preserve the structure of the microbial community being sequenced. Many studies have compared the effectiveness of preservative media for a variety of sample types (Rissanen et al., 2010; Vlčková et al., 2012; Gray, Pratte \& Kellogg, 2013; Tatangelo et al., 2014; Choo, Leong \& Rogers, 2015; Anderson et al., 2016; Blekhman et al., 2016; Amir et al., 2017;

Vogtmann et al., 2017), and typically conclude that most preservatives alter microbial community structure to a greater or lesser degree. Some preservatives such as ethanol and phenol-chloroform are also hazardous and therefore face travel restrictions, limiting their use for field collections. There is therefore an ongoing need to identify storage media that are effective, inexpensive, and well-suited to field collections.

Dimethyl sulfoxide (DMSO)-ethylenediamine tetraacetic acid (EDTA)-saturated salt solution (DESS, also known as SSD) is a non-proprietary, non-hazardous storage medium that shows strong potential for use as a preservative in microbial ecology studies. In this medium, DMSO permeates cells and facilitates the rapid entry of EDTA that suppresses nuclease activity by chelating divalent cations. Sodium chloride salt further suppresses enzymatic activity and contributes sodium ions that stabilize the negatively charged phosphate backbone of DNA (Seutin, White \& Boag, 1991). DESS was first used to preserve avian blood samples by Seutin, White \& Boag (1991) and has since been used to preserve the morphology and DNA of nematodes (Yoder et al., 2006), corals, and small marine invertebrates (Gaither et al., 2011). DESS did not strongly alter microbial community structure when used to preserve mock microbial communities created from environmental isolates (Gray, Pratte \& Kellogg, 2013) or soil and water samples, with and without cold storage (Tatangelo et al., 2014). Other DMSO-based preservatives have similar properties (Kerckhof et al., 2014). DESS therefore shows promise as a broadly applicable preservative that can overcome the fragility or lack of cold chains during sample transport between collection sites and the laboratory.

Here, we evaluated DESS as a preservative using the microbial communities found in fungus gardens grown by the ant Trachymyrmex septentrionalis as a model system studied by our research group. We typically collect these samples in hot and humid 
locations that are far from the lab, meaning that our cold chain is susceptible to failure. We further validated our field-based observations using a mock microbial community with a defined structure. Our results suggest that DESS is an excellent preservative of microbial community structure that is useful for field collections where cold transport and storage are challenging.

\section{MATERIALS AND METHODS}

\section{Sample collection}

Trachymyrmex septentrionalis colonies were collected in New Jersey, Florida, Georgia, and North Carolina during 2014 and 2015. Permits for collecting samples were obtained from the corresponding state department: State of New Jersey Department of Environmental Protection Division of Parks and Forestry State Park Service Unnumbered Letter of Authorization; North Carolina Division of Parks and Recreation Scientific Research and Collecting Permit 2015_0030; Florida Department of Agriculture and Consumer Services unnumbered Letter of Authorization; Georgia Department of Natural Resources State Parks \& Historic Sites Scientific Research and Collection Permit 032015; Georgia Department of Natural Resources Wildlife Resources Division unnumbered Letter of Authorization. T. septentrionalis colonies were identified by their distinctive "half-moon" mound shape and the presence of T. septentrionalis worker ants. An initial $\sim 25 \mathrm{~cm}$ deep trench was dug beside each colony entrance and then expanded until the fungus garden chamber was gently breached. After expanding this opening, the fungus garden was removed using a flame-sterilized spoon. Fungus gardens were naturally homogenized during collection by crumbling due to their fragility. Approximately $200 \mathrm{mg}$ of each fungus garden was subsampled into DESS (20\% DMSO (v/v), $250 \mathrm{mM}$ EDTA, saturated with sodium chloride), 15\% (v/v) glycerol, or PBS (137 mM NaCl, $2.7 \mathrm{mM} \mathrm{KCl}$, $10 \mathrm{mM} \mathrm{Na}_{2} \mathrm{HPO}_{4}, 2 \mathrm{mM} \mathrm{KH}_{2} \mathrm{PO}_{4}, \mathrm{pH}=7.4$ ), frozen immediately on dry ice, and then transferred to $-80{ }^{\circ} \mathrm{C}$ storage upon return to the laboratory. Glycerol was chosen for comparison based on its potential use as a cryoprotectant that would allow cells to be cultured upon return to the lab. PBS served as a baseline that was not expected to facilitate sample preservation beyond maintaining osmotic balance. The Atta texana colony used to generate data for Fig. S1 was collected from Louisiana in 2016 under Louisiana Department of Wildlife and Fisheries permit WL-Research-2016-10. This colony was already being maintained in the laboratory at the time of sampling. A small $(5 \times 5 \times 5 \mathrm{~cm})$ piece of the fungus garden was homogenized in a sterile petri dish and sampled in the same manner using DESS, PBS, and $100 \%$ ethanol. These samples were immediately placed in the $-80{ }^{\circ} \mathrm{C}$ freezer for 10 days prior to processing.

\section{Sample processing}

\section{Fungus gardens}

Samples were warmed to $-20{ }^{\circ} \mathrm{C}$ overnight and then thawed at $4{ }^{\circ} \mathrm{C}$ just prior to processing (2-5 hours). Replicate subsamples were pooled to obtain $\sim 1 \mathrm{~g}$ wet mass of fungus garden (equivalent to $250-500 \mathrm{mg}$ dry mass). We adapted the protocol of Apajalahti et al. (1998) to enrich for bacterial cells prior to DNA extraction, using medium speed vortexing 
to homogenize samples instead of rotational shaking and a final centrifugation at $15,000 \times \mathrm{g}$ for 15 min instead of $30,000 \times g$ to pellet bacterial cells. DNA was extracted from the resulting cell pellets using a CTAB/bead beating protocol modified from Cafaro \& Currie (2005) by using three 2-min cycles of bead beating (Biospec Minibeadbeater, Bartlesville, OK, USA) separated by 2.5 min cooling on ice for cell lysis and 24:1 chloroform:isoamyl alcohol for DNA extraction. DNA extracts were resuspended in nuclease-free water and quantified spectrophotometrically using a BioSpec EON plate reader with a Take3Trio plate. Negative DNA extraction controls that contained water instead of sample were included alongside each extraction batch. DNA extracts with $A_{260 / 280}$ or $A_{260 / 230}$ ratios $\leq 1.5$ were purified using Agencourt XP magnetic beads (Beckman Coulter, Brea, CA, USA) following the manufacturer's protocol.

\section{Mock community}

A ZymoBIOMICS Microbial Community Standard (Zymo Research Corporation, Irvine, CA, USA) composed of five Firmicutes (Gram positive) and three Proteobacteria (Gram negative) was used to precisely test DESS's ability to preserve microbiome DNA. Although not as diverse as T. septentrionalis fungus gardens, the major constituents of the mock community belong to the predominant phyla that we observed in the T. septentrionalis fungus gardens (see Results). One vial of this mock community was divided into 18 equal aliquots and each aliquot was centrifuged at $5,000 \times g$ for 5 min to pellet the cells. The supernatant containing the proprietary Zymo preservative was removed and the cells were resuspended in one ml of DESS, PBS, or 15\% glycerol by vortexing. DNA was extracted from two samples resuspended in each preservative as described above either immediately (before freezing; $t_{0}$ ) or after one $\left(t_{1}\right)$ or two $\left(t_{2}\right)$ months of storage at $-80{ }^{\circ} \mathrm{C}$. These DNA extracts were quality checked as described above but none required magnetic bead cleaning. Negative controls were not used for this experiment because of the known composition of the mock communities.

\section{PCR screening}

All quality-checked DNA extracts were screened by PCR amplification of the V4 region of the $16 \mathrm{~S}$ rRNA gene before sequencing on an Illumina MiSeq. Each $25 \mu \mathrm{l}$ reaction used $1 X$ GoTaq reaction buffer (Promega, Madison, WI, USA), $0.3 \mu \mathrm{M}$ each of primers 515F and 806R (Caporaso et al., 2011; Invitrogen, Carlsbad, CA, USA), 1.25 units of GoTaq DNA polymerase (Promega, Madison, WI, USA), $300 \mathrm{ng} / \mu \mathrm{l}$ bovine serum albumin (New England BioLabs, Ipswich, MA, USA), $50 \mathrm{ng}$ of template DNA, and nuclease-free water (Thomas Scientific, Swedesboro, NJ, USA). PCR reactions were run on a T-100 Thermal Cycler (BioRad, Hercules, CA, USA) for $3 \mathrm{~min}$ at $95^{\circ} \mathrm{C}, 30$ cycles of: $30 \mathrm{~s}$ at $95^{\circ} \mathrm{C}, 30 \mathrm{~s}$ at $50{ }^{\circ} \mathrm{C}$, and $60 \mathrm{~s}$ at $72{ }^{\circ} \mathrm{C}$, followed by a single $5 \mathrm{~min}$ cycle at $72{ }^{\circ} \mathrm{C}$. Bands were visualized using agarose gel electrophoresis. PCR reactions lacking the expected $350 \mathrm{bp}$ product were re-cleaned using magnetic beads as described above and screened by PCR a second time.

\section{Community amplicon sequencing}

Quality-checked, PCR-screened DNA extracts were submitted to the University of Connecticut Microbial Analysis, Resources, and Services (MARS) facility for sequencing. 
Submitted DNA samples were quantified fluorometrically using a PicoGreen (Invitrogen, Carlsbad, CA, USA) assay in 384-well plates read on a Synergy plate reader (BioTek, Winooski, VT, USA). After quantification, $30 \mathrm{ng}$ of sample DNA was added to $1 \times$ Phusion High Fidelity master mix (New England Biolabs, Ipswich, MA, USA) containing $1 \mu \mathrm{M}$ indexed sequencing primers with Illumina adapters (Kozich et al., 2013; Invitrogen, Carlsbad, CA, USA), four nM non-Illumina primers (515F/806R; Caporaso et al., 2011; Invitrogen, Carlsbad, CA, USA) to a final volume of $50 \mu$. These reactions were split into three equal aliquots and PCR amplified using settings: $94{ }^{\circ} \mathrm{C}$ for $3 \mathrm{~min}$ initial denaturation, 30 cycles of $94{ }^{\circ} \mathrm{C}$ for $45 \mathrm{~s}$ denaturation, $50{ }^{\circ} \mathrm{C}$ for 1 -min annealing, $72{ }^{\circ} \mathrm{C}$ for $1.5 \mathrm{~min}$ extension, and $72{ }^{\circ} \mathrm{C}$ for $10 \mathrm{~min}$ final extension. These PCR reactions were re-pooled and quantified using a QIAxcel instrument (Qiagen, Venlo, Netherlands). Sample libraries with a PCR product concentration $>0.5 \mathrm{ng} / \mu \mathrm{l}$ and peak(s) at $400 \mathrm{bp}$ $( \pm 15 \%)$ were pooled by adding equal masses of PCR product from each sample. These pooled libraries were cleaned using Mag-Bind RXNPure Plus beads (OMEGA bio-tek, Norcross, GA, USA), resuspended in $25 \mu \mathrm{l}$ molecular biology grade water, quantified using a Qubit assay (Invitrogen, Carlsbad, CA, USA), and adjusted to $4 \mathrm{nM}$ (1.5 ng/ $\mu \mathrm{l}$ when using MARS adaptors). Amplicon libraries were diluted to $6 \mathrm{pM}$ in Illumina HT1 buffer with $30 \%$ PhiX phage DNA added and sequenced using a V2 $(2 \times 250)$ cartridge on an Illumina MiSeq instrument.

\section{Bioinformatic analysis}

16S rRNA gene sequences were processed in R v3.4.3 (R Development Core Team, 2017) using the DADA2 v1.7.0 pipeline (Callahan et al., 2016) following the guidelines at https://benjjneb.github.io/dada2/tutorial.html (accessed 7/2/2018). Processed sequences and accompanying metadata were imported into phyloseq v1.22.3 (McMurdie \& Holmes, 2013) and screened for potential contaminants using decontam v0.20.0 (Davis et al., 2018) using the decontam "prevalence protocol" with the $P^{*}$ threshold set to 0.5. Amplicon sequence variants (ASVs) that were not classified as bacteria were removed, as were bacterial sequences that were not classified to at least the phylum level.

Decontam identified 36 fungus garden ASVs as contaminants (ranging from $0 \%$ to $10.2 \%$ of the sequences in each sample, mean $1.4 \%$ ), which were removed from this dataset. After excluding four samples with low read counts, the final fungus garden dataset contained 26 samples from 10 T. septentrionalis colonies (minimum: 11,379 reads; mean: 46,125 reads). Instead of using decontam, seven ASVs (ranging from $0.4 \%$ to $3.4 \%$ of the sequences in each sample, mean 1.6\%) were manually detected and removed from the mock community dataset because they did not match known members of that community. All 16 mock community samples were included in the final mock community dataset (minimum: 24,891 reads; mean: 36,445 reads). The raw sequence data have been deposited in the National Center for Biotechnology Information database under BioProject ID PRJNA479679.

The resulting phyloseq-compatible $\mathrm{R}$ data object was subsampled to the lowest read count among all libraries and ASV counts were converted to relative abundances. Weighted UniFrac distances were calculated, ordinated, and visualized using the distance, 
ordinate, and plot_ordination functions in phyloseq, respectively. Phyloseq was also used to calculate Shannon's diversity, Chaol diversity, and the observed diversity metrics using the estimate_richness function. Permutational analysis of variance (PERMANOVA) tests were done using Adonis in the vegan R package v2.4-5 (Oksanen et al., 2017). The aov, kruskal.test, and TukeyHSD commands implemented in the base R package were used to perform analysis of variance (ANOVA), Kruskal-Wallace and Tukey's honest significant difference testing, respectively. The code and metadata files to reproduce these analyses are available in File S1.

\section{RESULTS}

\section{Ant fungus gardens}

In earlier work sequencing $16 \mathrm{~S}$ rRNA genes in T. septentrionalis fungus gardens, Ishak et al. (2011) reported difficulty in generating sequencing reads from ethanolpreserved fungus garden samples. Similarly, our extractions of fungus gardens preserved in ethanol consistently yielded low-quality DNA (see Fig. S1, DNA extractions from A. texana fungus gardens). DNA extracts from ethanol-preserved fungus garden samples had a green-brown discoloration when compared to either PBS- or DESS-preserved samples, which were both visibly clear (Fig. S1A). Ethanol-preserved samples yielded more DNA than DESS- or PBS-preserved samples, but this DNA was highly fragmented (Fig. S1B). PCR amplification of the 16S rRNA gene V4 region from these ethanolpreserved samples was unsuccessful (Fig. S1C). Our results agreed with previous work (Rissanen et al., 2010; Gaither et al., 2011) that indicated poor preservation of DNA when stored in ethanol. We therefore discontinued the use of ethanol as a preservative for our microbiome characterization studies and instead focused on DESS as a safe and inexpensive alternative to ethanol for preservation of microbial community DNA during our field work.

To evaluate DESS as a preservative of microbial community DNA, we compared T. septentrionalis fungus garden samples stored in DESS to parallel samples stored in either PBS as a reference control or glycerol as a cryoprotectant that was not expected to prevent DNA degradation. Compared to between-colony differences, the type of preservative in which samples were stored only minimally correlated with variation in microbial community structure. Principal coordinate analysis (PCoA) of both Weighted and Unweighted UniFrac distances showed that samples from the same colony group together. In contrast, samples stored in different media are distributed throughout each PCoA plot (Fig. 1B-Weighted UniFrac, Fig. S2-Unweighted UniFrac). Colony of origin accounted for the largest amount of the between-sample variation in $\beta$-diversity (PERMANOVA Weighted UniFrac, $F=52.496, R^{2}=0.799, p=0.001$, Unweighted UniFrac $F=2.2136, R^{2}=0.555, p=0.001$ ) with storage medium accounting for a very small percentage of this variation in the Weighted but not the Unweighted Unifrac analysis (PERMANOVA Weighted UniFrac, $F=11.78, R^{2}=0.039, p=0.006$, Unweighted UniFrac $\left.F=0.84939, R^{2}=0.069, p=0.764\right)$ that was linked to colony of origin (colony-by-storage medium interaction: PERMANOVA Weighted UniFrac, $F=8.37$, $R^{2}=0.156, p=0.010$, Unweighted UniFrac $F=1.0959, R^{2}=0.311, p=0.317$ ). 

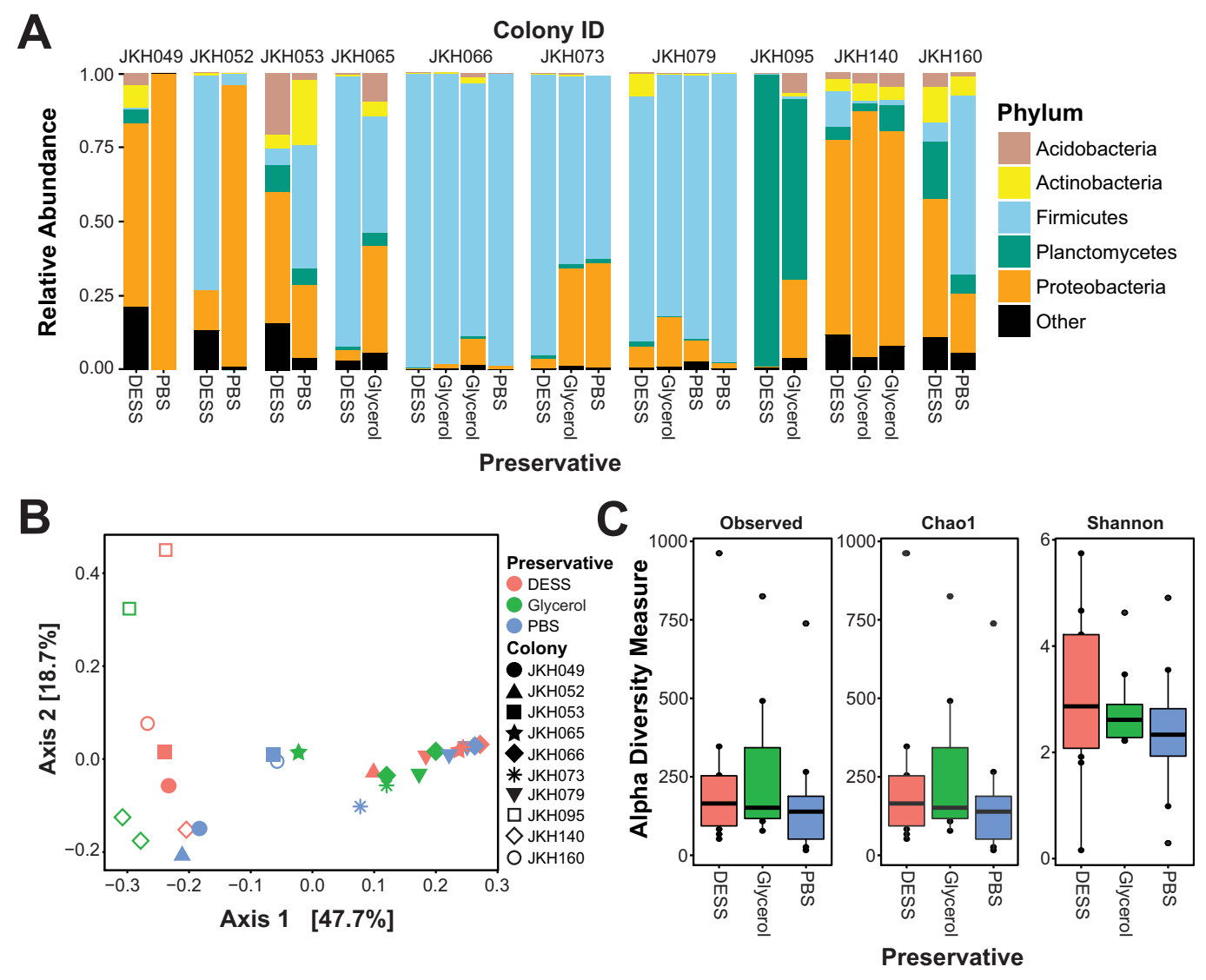

Figure 1 Preservative type does not alter the community structure of $T$. septentrionalis fungus gardens. (A) Relative abundances of phyla in T. septentrionalis fungus gardens, grouped by colony. Phyla with relative abundances $\leq 15 \%$ in all samples are grouped into a single "Other" category. (B) PCoA of Weighted UniFrac distances between T. septentrionalis fungus garden bacterial communities. Colors indicate preservative types, and shapes indicate samples from different colonies. (C) Alpha-diversity (Shannon's, Observed, and Chaol) in T. septentrionalis fungus gardens. Center bars in the box plot indicate the median, top and bottom of boxes indicate the 25th and 75th quartile, respectively, and whiskers indicate \pm 1.5 times the standard error. Single points represent outliers.

Full-size $\underset{0}{0}$ DOI: 10.7717/peerj.6414/fig-1

Weighted UniFrac distances between samples from the same colony that were stored in different preservatives did not significantly differ from each other (Kruskal-Wallace, $\mathrm{D} f=2, \chi^{2}=4.779, p=0.090$ ), implying that one preservative type did not alter microbial community structure more than the others. Like $\beta$-diversity, the $\alpha$-diversity of samples collected from the same colony did not vary between preservative types (Fig. 1C; Kruskal-Wallace, Shannon: $\mathrm{D} f=2, \chi^{2}=0.934, p=0.627$; Chaol and Observed: $\mathrm{D} f=2$, $\left.\chi^{2}=1.127, p=0.569\right)$. Collectively, these data indicate that preservative type had at most a minor effect on the observed structure of T. septentrionalis fungus garden microbial communities.

\section{Mock community samples}

Our experiments with $T$. septentrionalis fungus gardens suggested that preservative type may have a small effect on community structure. However, the lack of a reference standard 

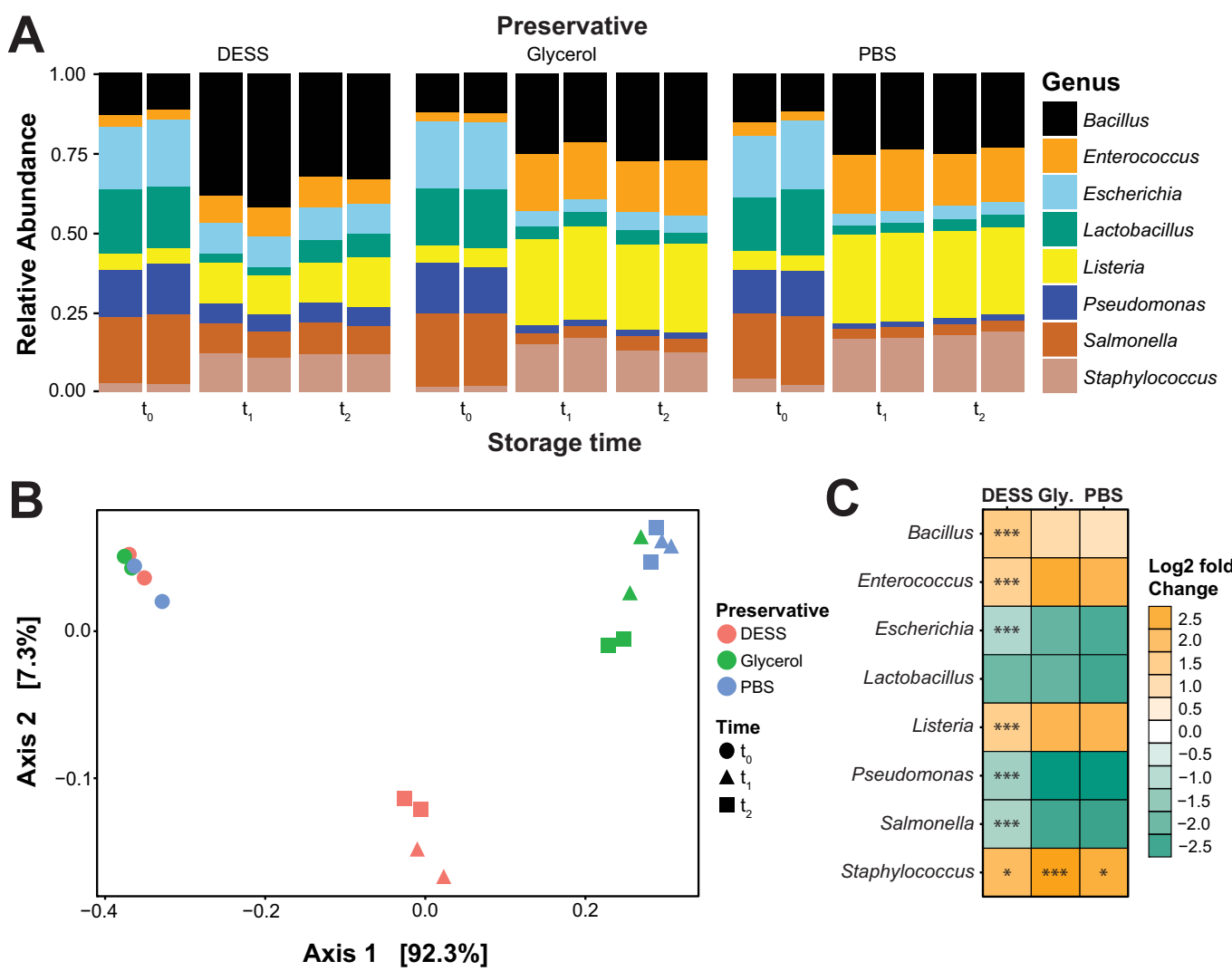

Figure 2 DESS preserves microbial mock community structure better than PBS or glycerol. (A) Relative abundances of genera in mock community samples. (B) PCoA of Weighted UniFrac distances between mock communities stored in DESS, PBS, or glycerol, indicated by different colors, for 0,1 , or 2 months, indicated by different shapes. (C) Heatmap showing $\log 2$ fold changes in relative abundance of genera in the mock community compared to $t_{0}$ samples. ${ }^{*}$ Indicate where these changes were significantly different between preservatives (Bonferroni corrected $p$-values: ${ }^{*} p<0.01,{ }^{* * *} p<0.001$ ).

Full-size $\boldsymbol{0}$ DOI: $10.7717 /$ peerj.6414/fig-2

prevented us from determining which samples best represented the true structure of T. septentrionalis fungus garden microbial communities and which represented artificial community structures that were biased by the effects of storage. We therefore used a mock community of known composition to isolate the effect of preservative on microbial community structure. Storage at $-80^{\circ} \mathrm{C}$ for 1 or 2 months changed the relative abundances of all taxa in all preservatives when compared to the corresponding unfrozen $\left(t_{0}\right)$ samples (Fig. 2A). The PCoA of the Weighted UniFrac distances showed three distinct clusters, containing either: (1) all $t_{0}$ samples; (2) $t_{1}$ and $t_{2}$ DESS samples; or (3) $t_{1}$ and $t_{2}$ glycerol and PBS samples (Fig. 2B). As with fungus garden samples, variation in mock community structure correlated minimally with preservative type (PERMANOVA, $F_{(2,17)}=163.8$, $\left.R^{2}=0.133, p=0.001\right)$. Instead, variation in mock community structure was highly correlated to storage time (PERMANOVA, $F_{(2,17)}=977.5, R^{2}=0.794, p=0.010$ ). The mean Weighted UniFrac distances between $t_{1}$ and $t_{2}$ samples frozen in the same preservative were very small $(\mathrm{DESS}=0.054$, glycerol $=0.055, \mathrm{PBS}=0.036)$ and not significantly different from each other (ANOVA, $\left.F_{(2,18)}=1.959, p=0.175\right)$. These small 
distances imply that "time" actually represents changes induced by freezing and/or any amount of frozen storage.

Mock community samples frozen in DESS changed less during storage at $-80{ }^{\circ} \mathrm{C}$ compared to the samples that were stored in either PBS or glycerol (Fig. 2C). Bacillus, Enterococcus, Listeria, and Staphylococcus were all overrepresented in mock communities stored in all preservatives relative to the $t_{0}$ baseline samples. Similarly, Escherichia, Lactobacillus, Pseudomonas, and Salmonella were all underrepresented in mock communities stored in all preservatives relative to the $t_{0}$ baseline samples. The relative abundance of six out of eight taxa differed from the $t_{0}$ baseline samples less when frozen in DESS compared to glycerol and PBS (Fig. 2C). The exceptions were Lactobacillus, whose relative abundance changed equally in all preservatives, and Bacillus, whose relative abundance changed more in DESS compared to the glycerol and PBS. Changes in taxon relative abundance compared to the $t_{0}$ baseline did not differ between taxa stored in glycerol or PBS (Tukey's Honest Significance with Bonferroni correction, $p>0.050$ ), except for Staphylococcus (Tukey's Honest Significance with Bonferroni correction, $p=0.016)$. Collectively, these data show that although freezing changes the structure of all mock community samples, communities stored in DESS were more similar to that of unfrozen $t_{0}$ baseline samples compared to samples stored in glycerol or PBS.

\section{DISCUSSION}

Trachymyrmex septentrionalis fungus garden microbial communities were more diverse than what has been reported for fungus gardens from several related ant species.

Fungus-growing ants from the genera Atta and Acromyrmex raise fungus gardens that contain low microbial diversity and that are dominated by Gammaproteobacteria, as are fungus gardens grown by T. zeteki (Suen et al., 2010; Aylward et al., 2012, 2014). In contrast, our results suggest that $T$. septentrionalis fungus garden microbial communities are highly variable (Fig. 1A). Of the 10 sampled fungus gardens, four were dominated by Firmicutes, two were dominated by Proteobacteria, and one was dominated by Planctomycetes. The remaining three colonies had high amounts of both Firmicutes and Proteobacteria (Fig. 1A). Acidobacteria, Actinobacteria, Verrucomicrobia, and (except for colony JKH095) Planctomycetes had low abundances in most fungus gardens. Our results agree with the limited data available for other T. septentrionalis fungus gardens (Ishak et al., 2011) and some other ant genera (Kellner et al., 2015). These differences between T. septentrionalis and leaf-cutting ant fungus gardens could be due to species-specific differences in habitat and foraging behavior (De Fine Licht \& Boomsma, 2010) that might lead to different microbes entering fungus gardens via different substrates. Although our limited dataset lacks sufficient statistical power to robustly identify ecological factors that structure T. septentrionalis fungus garden microbial communities, these aspects remain active and ongoing areas of research in our lab.

Preservative type had only a small impact on T. septentrionalis fungus garden microbial community structure. The $\sim 4 \%$ of variation in fungus garden microbial community structure attributed to preservative by PERMANOVA was dwarfed by the $>75 \%$ of 
variation attributed to the colony of origin (Fig. 1B). Preservative type also did not correlate with changes in the alpha-diversity of $T$. septentrionalis fungus garden microbial communities (Fig. 1C). This minimal effect of preservative parallels other studies where between-host differences in microbial community structure greatly exceeded differences caused by variation in storage strategy (Bai et al., 2012; Hill et al., 2016; Song et al., 2016).

Because our T. septentrionalis fungus gardens were all frozen in the field, we lacked a reference community against which to compare the slightly different microbial community structures of the fungus gardens stored in DESS compared to those stored in glycerol or PBS. We therefore used a mock community to more precisely assess changes in microbial community structure caused by each preservative. In these experiments, all mock communities changed relative to $t_{0}$ samples, likely indicating either a consistently negative effect of storage for $1-2$ months at $-80{ }^{\circ} \mathrm{C}$ (Bahl, Bergström \& Licht, 2012; Kia et al., 2016) and/or the consistently negative effect of the additional freeze/thaw cycle undergone by the $t_{1}$ and $t_{2}$ samples but not the $t_{0}$ samples (Cardona et al., 2012; Gorzelak et al., 2015). Future research into DESS should focus on how microbial community structure changes under fluctuating storage conditions, as might occur in a broken cold chain. Four of the five Firmicutes strains in the mock community were overrepresented after storage at $-80{ }^{\circ} \mathrm{C}$. Such increases in Firmicutes following cold storage has been reported previously (Bahl, Bergström \& Licht, 2012; Anderson et al., 2016; Hill et al., 2016; Song et al., 2016). However, these changes were less severe for samples stored in DESS compared to those stored in glycerol or PBS, complementing previous studies that showed DESS to be an effective storage medium using different sample types and storage temperatures (Gray, Pratte \& Kellogg, 2013; Tatangelo et al., 2014).

\section{CONCLUSIONS}

In summary, our results suggest that DESS is an excellent storage medium for microbial ecology samples. Storage in DESS did not obscure the ecological differences between the microbial communities of fungus gardens collected from different ant colonies. DESS also preserved the community structure of a mock community more faithfully than either glycerol or PBS. DESS is inexpensive and non-hazardous, making it easy to transport. Together, these attributes suggest that DESS is a versatile and economical preservative that is suitable for the transport and storage of microbial communities.

\section{ACKNOWLEDGEMENTS}

We thank the members of the Klassen lab for assistance with sampling, and the University of Connecticut MARS facility for assistance with library preparation and DNA sequencing. We would also like to thank the personnel from the many state forests and parks from which we collected ants for their valuable insights and enthusiasm for the scientific research conducted in their forests and parks, and the anonymous reviewers for their constructive feedback on this manuscript. 


\section{ADDITIONAL INFORMATION AND DECLARATIONS}

\section{Funding}

This work was supported by the US National Science Foundation (IOS-1656475) and the University of Connecticut. The funders had no role in study design, data collection and analysis, decision to publish, or preparation of the manuscript.

\section{Grant Disclosures}

The following grant information was disclosed by the authors:

US National Science Foundation: IOS-1656475.

University of Connecticut.

\section{Competing Interests}

The authors declare that they have no competing interests.

\section{Author Contributions}

- Kevin M. Lee conceived and designed the experiments, performed the experiments, analyzed the data, contributed reagents/materials/analysis tools, prepared figures and/or tables, authored or reviewed drafts of the paper, approved the final draft.

- Madison Adams conceived and designed the experiments, performed the experiments, contributed reagents/materials/analysis tools, approved the final draft.

- Jonathan L. Klassen conceived and designed the experiments, analyzed the data, prepared figures and/or tables, authored or reviewed drafts of the paper, approved the final draft.

\section{Field Study Permissions}

The following information was supplied relating to field study approvals (i.e., approving body and any reference numbers):

Permits for collecting samples were obtained from the corresponding state department: State of New Jersey Department of Environmental Protection Division of Parks and Forestry State Park Service Unnumbered Letter of Authorization; North Carolina Division of Parks and Recreation Scientific Research and Collecting Permit 2015_0030;

Florida Department of Agriculture and Consumer Services unnumbered Letter of Authorization; Georgia Department of Natural Resources State Parks \& Historic Sites Scientific Research and Collection Permit 032015; Georgia Department of Natural Resources Wildlife Resources Division unnumbered Letter of Authorization; Louisiana Department of Wildlife and Fisheries permit WL-Research-2016-10.

\section{DNA Deposition}

The following information was supplied regarding the deposition of DNA sequences:

The raw sequence data are available at NCBI BioProject under BioProject ID PRJNA479679.

\section{Data Availability}

The following information was supplied regarding data availability:

The raw data (code and metadata files) are available in the Supplemental Files. 


\section{Supplemental Information}

Supplemental information for this article can be found online at http://dx.doi.org/10.7717/ peerj.6414\#supplemental-information.

\section{REFERENCES}

Amir A, McDonald D, Navas-Molina JA, Debelius J, Morton JT, Hyde E, Robbins-Pianka A, Knight R. 2017. Correcting for microbial blooms in fecal samples during room-temperature shipping. mSystems 2(2):e00199-16 DOI 10.1128/mSystems.00199-16.

Anderson EL, Li W, Klitgord N, Highlander SK, Dayrit M, Seguritan V, Yooseph S, Biggs W, Venter JC, Nelson KE, Jones MB. 2016. A robust ambient temperature collection and stabilization strategy: enabling worldwide functional studies of the human microbiome. Scientific Reports 6(1):31731 DOI 10.1038/srep31731.

Apajalahti JH, Särkilahti LK, Mäki BR, Heikkinen JP, Nurminen PH, Holben WE. 1998. Effective recovery of bacterial DNA and percent-guanine-plus-cytosine-based analysis of community structure in the gastrointestinal tract of broiler chickens. Applied and Environmental Microbiology 64:4084-4088 DOI 10.1128/AEM.71.7.3911-3916.2005.

Aylward FO, Burnum KE, Scott JJ, Suen G, Tringe SG, Adams SM, Barry KW, Nicora CD, Piehowski PD, Purvine SO, Starrett GJ, Goodwin LA, Smith RD, Lipton MS, Currie CR. 2012. Metagenomic and metaproteomic insights into bacterial communities in leaf-cutter ant fungus gardens. ISME Journal 6(9):1688-1701 DOI 10.1038/ismej.2012.10.

Aylward FO, Suen G, Biedermann PHW, Adams AS, Scott JJ, Malfatti SA, Del Rio TG, Tringe SG, Poulsen M, Raffa KF, Klepzig KD, Currie CR. 2014. Convergent bacterial microbiotas in the fungal agricultural systems of insects. mBio 5(6):e02077-14 DOI 10.1128/mBio.02077-14.

Bahl MI, Bergström A, Licht TR. 2012. Freezing fecal samples prior to DNA extraction affects the Firmicutes to Bacteroidetes ratio determined by downstream quantitative PCR analysis. FEMS Microbiology Letters 329(2):193-197 DOI 10.1111/j.1574-6968.2012.02523.x.

Bai G, Gajer P, Nandy M, Ma B, Yang H, Sakamoto J, Blanchard MH, Ravel J, Brotman RM. 2012. Comparison of storage conditions for human vaginal microbiome studies. PLOS ONE 7(5):e36934 DOI 10.1371/journal.pone.0036934.

Blekhman R, Tang K, Archie EA, Barreiro LB, Johnson ZP, Wilson ME, Kohn J, Yuan ML, Gesquiere L, Grieneisen LE, Tung J. 2016. Common methods for fecal sample storage in field studies yield consistent signatures of individual identity in microbiome sequencing data. Scientific Reports 6(1):31519 DOI 10.1038/srep31519.

Cafaro MJ, Currie CR. 2005. Phylogenetic analysis of mutualistic filamentous bacteria associated with fungus-growing ants. Canadian Journal of Microbiology 51(6):441-446 DOI 10.1139/w05-023.

Callahan BJ, McMurdie PJ, Rosen MJ, Han AW, Johnson AJA, Holmes SP. 2016. DADA2: high-resolution sample inference from Illumina amplicon data. Nature Methods 13(7):581-583 DOI 10.1038/nmeth.3869.

Caporaso JG, Lauber CL, Walters WA, Berg-Lyons D, Lozupone CA, Turnbaugh PJ, Fierer N, Knight R. 2011. Global patterns of $16 \mathrm{~S}$ rRNA diversity at a depth of millions of sequences per sample. Proceedings of the National Academy of Sciences of the United States of America 108(Suppl 1):4516-4522 DOI 10.1073/pnas.1000080107.

Cardona S, Eck A, Cassellas M, Gallart M, Alastrue C, Dore J, Azpiroz F, Roca J, Guarner F, Manichanh C. 2012. Storage conditions of intestinal microbiota matter in metagenomic analysis. BMC Microbiology 12(1):1471-2180 DOI 10.1186/1471-2180-12-158. 
Choo JM, Leong LEX, Rogers GB. 2015. Sample storage conditions significantly influence faecal microbiome profiles. Scientific Reports 5(1):16350 DOI 10.1038/srep16350.

Davis NM, Proctor D, Holmes SP, Relman DA, Benjamin J. 2018. Simple statistical identification and removal of contaminant sequences in marker-gene and metagenomics data. Microbiome 6(1):226 DOI 10.1186/s40168-018-0605-2.

De Fine Licht HH, Boomsma JJ. 2010. Forage collection, substrate preparation, and diet composition in fungus-growing ants. Ecological Entomology 35(3):259-269 DOI 10.1111/j.1365-2311.2010.01193.x.

Gaither MR, Szabó Z, Crepeau MW, Bird CE, Toonen RJ. 2011. Preservation of corals in salt-saturated DMSO buffer is superior to ethanol for PCR experiments. Coral Reefs 30(2):329-333 DOI 10.1007/s00338-010-0687-1.

Gorzelak MA, Gill SK, Tasnim N, Ahmadi-Vand Z, Jay M, Gibson DL. 2015. Methods for improving human gut microbiome data by reducing variability through sample processing and storage of stool. PLOS ONE 10(9):e0134802 DOI 10.1371/journal.pone.0134802.

Gray MA, Pratte ZA, Kellogg CA. 2013. Comparison of DNA preservation methods for environmental bacterial community samples. FEMS Microbiology Ecology 83(2):468-477 DOI 10.1111/1574-6941.12008.

Hale VL, Tan CL, Niu K, Yang Y, Cui D, Zhao H, Knight R, Amato KR. 2016. Effects of field conditions on fecal microbiota. Journal of Microbiological Methods 130:180-188 DOI 10.1016/j.mimet.2016.09.017.

Hill CJ, Brown JRM, Lynch DB, Jeffery IB, Ryan CA, Ross RP, Stanton C, O'Toole PW. 2016. Effect of room temperature transport vials on DNA quality and phylogenetic composition of faecal microbiota of elderly adults and infants. Microbiome 4(1):19 DOI 10.1186/s40168-016-0164-3.

Hugerth LW, Andersson AF. 2017. Analysing microbial community composition through amplicon sequencing: from sampling to hypothesis testing. Frontiers in Microbiology 8:1561 DOI 10.3389/fmicb.2017.01561.

Ishak HD, Miller JL, Sen R, Dowd SE, Meyer E, Mueller UG. 2011. Microbiomes of ant castes implicate new microbial roles in the fungus-growing ant Trachymyrmex septentrionalis. Scientific Reports 1(1):00204 DOI 10.1038/srep00204.

Kellner K, Ishak HD, Linksvayer TA, Mueller UG. 2015. Bacterial community composition and diversity in an ancestral ant fungus symbiosis. FEMS Microbiology Ecology 91(7):fiv073 DOI 10.1093/femsec/fiv073.

Kerckhof FM, Courtens ENP, Geirnaert A, Hoefman S, Ho A, Vilchez-Vargas R, Pieper DH, Jauregui R, Vlaeminck SE, Van De Wiele T, Vandamme P, Heylen K, Boon N. 2014. Optimized cryopreservation of mixed microbial communities for conserved functionality and diversity. PLOS ONE 9(6):e99517 DOI 10.1371/journal.pone.0099517.

Kia E, MacKenzie BW, Middleton D, Lau A, Waite DW, Lewis G, Chan YK, Silvestre M, Cooper GJS, Poppitt SD, Taylor MW. 2016. Integrity of the human faecal microbiota following long-term sample storage. PLOS ONE 11(10):e0163666 DOI 10.1371/journal.pone.0163666.

Kozich JJ, Westcott SL, Baxter NT, Highlander SK, Schloss PD. 2013. Development of a dual-index sequencing strategy and curation pipeline for analyzing amplicon sequence data on the miseq illumina sequencing platform. Applied and Environmental Microbiology 79(17):5112-5120 DOI 10.1128/AEM.01043-13.

McMurdie PJ, Holmes S. 2013. Phyloseq: an R Package for reproducible interactive analysis and graphics of microbiome census data. PLOS ONE 8(4):e61217

DOI 10.1371/journal.pone.0061217. 
Oksanen J, Blanchet FG, Friendly M, Kindt R, Legendre P, McGlinn D, Minchin PR, O'Hara RB, Simpson GL, Solymos P, Stevens MHH, Szoecs E, Wagner H. 2017. vegan: community ecology package. $\mathrm{R}$ package version $2.4-5$. Available at https://CRAN.R-project.org/package:vegan.

Pollock J, Glendinning L, Wisedchanwet T, Watson M. 2018. The madness of microbiome: Attempting to find consensus "best practice" for $16 \mathrm{~S}$ microbiome studies. Applied and Environmental Microbiology 84(7):e02627-17 DOI 10.1128/AEM.02627-17.

R Development Core Team. 2017. $R$ : a language and environment for statistical computing. Vienna: The R Foundation for Statistical Computing. Available at http://www.R-project.org/.

Rissanen AJ, Kurhela E, Aho T, Oittinen T, Tiirola M. 2010. Storage of environmental samples for guaranteeing nucleic acid yields for molecular microbiological studies. Applied Microbiology and Biotechnology 88(4):977-984 DOI 10.1007/s00253-010-2838-2.

Rochelle PA, Cragg BA, Fry JC, John Parkes R, Weightman AJ. 1994. Effect of sample handling on estimation of bacterial diversity in marine sediments by $16 \mathrm{~S}$ rRNA gene sequence analysis. FEMS Microbiology Ecology 15(1-2):215-225 DOI 10.1111/j.1574-6941.1994.tb00245.x.

Seutin G, White BN, Boag PT. 1991. Preservation of avian blood and tissue samples for DNA analyses. Canadian Journal of Zoology 69(1):82-90 DOI 10.1139/z91-013.

Song SJ, Amir A, Metcalf JL, Amato KR, Xu ZZ, Humphrey G, Knight R. 2016. Preservation methods differ in fecal microbiome stability, affecting suitability for field studies. mSystems 1(3):e0002116 DOI 10.1128/mSystems.00021-16.

Suen G, Scott JJ, Aylward FO, Adams SM, Tringe SG, Pinto-Tomás AA, Foster CE, Pauly M, Weimer PJ, Barry KW, Goodwin LA, Bouffard P, Li L, Osterberger J, Harkins TT, Slater SC, Donohue TJ, Currie CR. 2010. An insect herbivore microbiome with high plant biomass-degrading capacity. PLOS Genetics 6(9):e1001129 DOI 10.1371/journal.pgen.1001129.

Tatangelo V, Franzetti A, Gandolfi I, Bestetti G, Ambrosini R. 2014. Effect of preservation method on the assessment of bacterial community structure in soil and water samples. FEMS Microbiology Letters 356(1):32-38 DOI 10.1111/1574-6968.12475.

Vandeputte D, Tito RY, Vanleeuwen R, Falony G, Raes J. 2017. Practical considerations for large-scale gut microbiome studies. FEMS Microbiology Reviews 41:S154-S167 DOI 10.1093/femsre/fux027.

Vlčková K, Mrázek J, Kopečný J, Petrželková KJ. 2012. Evaluation of different storage methods to characterize the fecal bacterial communities of captive western lowland gorillas (Gorilla gorilla gorilla). Journal of Microbiological Methods 91(1):45-51 DOI 10.1016/j.mimet.2012.07.015.

Vogtmann E, Chen J, Amir A, Shi J, Abnet CC, Nelson H, Knight R, Chia N, Sinha R. 2017. Comparison of collection methods for fecal samples in microbiome studies. American Journal of Epidemiology 185(2):115-123 DOI 10.1093/aje/kww177.

Williamson KE, Kan J, Polson SW, Williamson SJ. 2011. Optimizing the indirect extraction of prokaryotic DNA from soils. Soil Biology and Biochemistry 43(4):736-748 DOI 10.1016/j.soilbio.2010.04.017.

Yoder M, Tandingan De Ley I, King IW, Mundo-Ocampo M, Mann J, Blaxter M, Poiras L, De Ley P. 2006. DESS: a versatile solution for preserving morphology and extractable DNA of nematodes. Nematology 8(3):367-376 DOI 10.1163/156854106778493448. 